\title{
Overall survival results from the randomized phase 2 study of palbociclib in combination with letrozole versus letrozole alone for first-line treatment of ER+/HER2- advanced breast cancer (PALOMA-1, TRI0-18)
}

\author{
Richard S. Finn ${ }^{1} \mathbb{D} \cdot$ Katalin Boer $^{2} \cdot$ Igor Bondarenko ${ }^{3} \cdot$ Ravindranath Patel $^{4} \cdot$ Tamas Pinter $^{5} \cdot$ Marcus Schmidt $^{6}$. \\ Yaroslav V. Shparyk $k^{7}$. Anu Thummala ${ }^{8} \cdot$ Nataliia Voitko $^{9} \cdot$ Eustratios Bananis $^{10} \cdot$ Lynn McRoy $^{10} \cdot$ Keith Wilner $^{11}$. \\ Xin Huang ${ }^{11}$. Sindy Kim ${ }^{11}$. Dennis J. Slamon ${ }^{1}$. Johannes Ettt ${ }^{12}$
}

Received: 31 March 2020 / Accepted: 13 June 2020 / Published online: 18 July 2020

(C) The Author(s) 2020

\begin{abstract}
Purpose Palbociclib is a cyclin-dependent kinase 4/6 (CDK4/6) inhibitor, approved in combination with endocrine therapy for the treatment of women and men with hormone receptor-positive, human epidermal growth factor receptor 2-negative advanced breast cancer (HR+/HER2- ABC). In the phase 2, open-label, PALOMA-1 trial, palbociclib plus letrozole significantly prolonged progression-free survival (PFS) versus letrozole alone (hazard ratio, 0.488; 95\% CI 0.319-0.748; $P=0.0004$; median PFS, 20.2 vs 10.2 months, respectively) in postmenopausal women with estrogen receptor-positive $(\mathrm{ER}+) / \mathrm{HER} 2-\mathrm{ABC}$. Here, we present the final overall survival (OS) and updated safety results.

Methods Postmenopausal women with ER+/HER2- ABC were randomized 1:1 to receive either palbociclib (125 mg/day, $3 / 1$ schedule) plus letrozole ( $2.5 \mathrm{mg} /$ day, continuous) or letrozole alone $(2.5 \mathrm{mg} / \mathrm{day}$, continuous). The primary endpoint was investigator-assessed PFS; secondary endpoints included OS and safety.

Results A total of 165 patients were randomized. At the data cutoff date of December 30, 2016 (median duration of follow-up, 64.7 months), the stratified hazard ratio for OS was 0.897 (95\% CI $0.623-1.294 ; P=0.281$ ); median OS in the palbociclib plus letrozole and letrozole alone arms was 37.5 and 34.5 months, respectively. The median time from randomization to first subsequent chemotherapy use was longer with palbociclib plus letrozole than letrozole alone (26.7 and 17.7 months, respectively). The most frequently reported adverse event in the palbociclib plus letrozole arm was neutropenia (any grade, $75 \%$; grade 3 or $4,59 \%$ ).

Conclusions Palbociclib plus letrozole treatment led to a numerical but not statistically significant improvement in median OS.

Pfizer Inc (NCT00721409)
\end{abstract}

Keywords Advanced breast cancer $\cdot$ ER+/HER2 $-\cdot$ Letrozole $\cdot$ Overall survival $\cdot$ Palbociclib

\section{Introduction}

Breast cancer is the most common type of cancer in women worldwide [1]; approximately $70 \%$ of breast cancers are hormone receptor-positive/human epidermal growth factor receptor 2-negative (HR+/HER2-) [2]. Although the long-term prognosis is good for patients whose disease

Richard S. Finn

RFinn@mednet.ucla.edu

Extended author information available on the last page of the article has not spread, historically, the 5-year survival rate for patients who develop or present with metastatic breast cancer is only approximately $25 \%$ [2, 3]. Single-agent endocrine therapy (ET; including antiestrogens and aromatase inhibitors [AIs]) had long been the mainstay of therapy for first-line treatment in postmenopausal women, with a better safety profile and quality of life compared with standard chemotherapy [4]. Recently, treatment with the cyclin-dependent kinase 4/6 (CDK4/6) inhibitor palbociclib in combination with endocrine therapy (ET) was incorporated in the National Comprehensive Cancer 
Network treatment guidelines for patients with $\mathrm{HR}+$ / HER2 - advanced breast cancer (ABC) [5].

Palbociclib is a first-in-class, potent, highly selective, orally administered, reversible CDK4/6 inhibitor [6]. Preclinical studies revealed that palbociclib in combination with ET preferentially and synergistically inhibited the cell cycle in human estrogen receptor-positive $(\mathrm{ER}+)$ breast cancer cell lines [7]. Based on these preclinical data, the phase 2 PALOMA-1 clinical trial was initiated to investigate the efficacy and safety of palbociclib plus ET as first-line treatment for postmenopausal women with ER+/HER2- ABC [8]. Progression-free survival (PFS) was the primary endpoint for this study. At the time of final analysis for PFS (data cutoff date, November 29, 2013), PFS was significantly prolonged in the palbociclib plus letrozole arm compared with the letrozole alone arm (hazard ratio, $0.488 ; 95 \%$ CI 0.319-0.748; 1-sided $P=0.0004$; median PFS, 20.2 vs 10.2 months, respectively) [8]. The results from this study led to the accelerated US Food and Drug Administration approval of palbociclib in combination with letrozole for the treatment of ER+/HER2- ABC in February 2015.

At the time of final PFS analysis, an interim overall survival (OS) analysis was performed. The hazard ratio was 0.813 (95\% CI $0.492-1.345$; 2 -sided $P=0.42$ ) [6]. A longer median OS was observed in the palbociclib plus letrozole arm compared with the letrozole alone arm (37.5 vs 33.3 months, respectively) [8]. Of note, the PALOMA-1 trial was not designed to perform formal hypothesis testing for OS due to the relatively small sample size; at the time of final PFS analysis, 30 (36\%) and 31 (38\%) OS events had occurred in the palbociclib plus letrozole and letrozole alone arms, respectively [8].

The phase 3 PALOMA-2 study confirmed the PFS benefit observed in PALOMA-1; PFS was significantly longer in the palbociclib plus letrozole arm compared with the placebo plus letrozole arm as first-line treatment in postmenopausal women with ER+/HER2- ABC (hazard ratio, 0.563; 95\% CI 0.461-0.687; $P<0.0001$; median PFS, 27.6 vs 14.5 months, respectively) [9]. In the phase 3 PALOMA-3 trial, PFS was also significantly longer in the palbociclib plus fulvestrant arm compared with the placebo plus fulvestrant arm in women with $\mathrm{HR}+$ / HER 2- ABC, regardless of menopausal status, whose disease had progressed on prior ET, in either the adjuvant or metastatic setting (hazard ratio, $0.497 ; 95 \%$ CI $0.398-0.620 ; P<0.0001$; median PFS, 11.2 vs 4.6 months, respectively) $[10,11]$. PALOMA-3 was the first phase 3 study of a CDK4/6 inhibitor to report OS results. The final OS analysis from PALOMA-3 showed longer median OS in the palbociclib plus fulvestrant arm compared with the placebo plus fulvestrant arm; however, this difference was not statistically significant (stratified hazard ratio, 0.81; 95\% CI $0.64-1.03 ; 2$-sided $P=0.09$; median OS, 34.9 vs
28.0 months, respectively) [10]. Here, we report the final OS and updated safety results from PALOMA-1, the first randomized study of a CDK4/6 inhibitor in $\mathrm{ABC}$ with the longest follow-up to date.

\section{Methods}

\section{Study design and patients}

Detailed methods for the phase 2 PALOMA-1 clinical trial have been previously published [8]. PALOMA-1 (NCT00721409) was an international, phase 2, open-label, multicenter, randomized clinical trial that enrolled postmenopausal women with ER+/HER2- ABC. Patients were enrolled into 2 cohorts that accrued sequentially. Patients were enrolled into cohort 1 based on ER+/HER2- status alone, whereas cohort 2 enrolled patients with tumors with cyclin D1 amplification, loss of p16, or both. After an interim analysis, accrual into cohort 2 was stopped, and the analysis plan was amended to combine both cohorts for the analyses of the study endpoints.

For both cohorts, randomization was stratified by disease site (visceral, bone only, or other) and disease-free interval (DFI; > 12 months from the end of adjuvant therapy to recurrence versus $\leq 12$ months from the end of adjuvant therapy to recurrence or de novo advanced disease). Key inclusion criteria were ER+/HER2 - tumors, women, aged $\geq 18$ years, postmenopausal status, Eastern Cooperative Oncology Group (ECOG) performance status 0 or 1, adequate organ function, and measurable disease per Response Evaluation Criteria in Solid Tumors (RECIST) criteria or bone-only disease. Key exclusion criteria were prior systemic treatment for advanced disease, prior treatment with (neo)adjuvant letrozole with disease recurrence $\leq 12$ months, and prior treatment with a CDK inhibitor.

\section{Treatment}

Patients were randomized 1:1 to receive either oral palbociclib $125 \mathrm{mg}$ /day, 3 weeks on treatment followed by 1 week off ( $3 / 1$ schedule) plus continuous oral letrozole $2.5 \mathrm{mg} / \mathrm{day}$ or continuous letrozole $2.5 \mathrm{mg} /$ day alone. Study treatment continued until disease progression, unacceptable toxicity, study withdrawal, or death. Palbociclib dose modifications, including cycle delay, dosing interruption, and dose reduction, were permitted to manage adverse events (AEs). No letrozole dose adjustment was allowed, but dosing interruptions were permitted. 


\section{Outcomes}

The primary study endpoint was investigator-assessed PFS, defined as the time from randomization to the date of first documentation of objective progression (based on RECIST v.1.0) or death due to any cause. Secondary endpoints included OS, objective response, clinical benefit rate, duration of response, and safety.

\section{Statistical analysis}

Overall survival was defined as the time from randomization date to the date of death due to any cause. OS was assessed in the intention-to-treat population, defined as all randomized patients, and in subgroups based on baseline demographic and disease characteristics using the Kaplan-Meier method; log-rank tests were used to compare OS between treatment arms. Cox regression models were used to estimate the treatment hazard ratio and associated $95 \%$ CI. The time to first use of subsequent chemotherapy was also assessed using the Kaplan-Meier method. Safety was evaluated in the astreated population, defined as all patients treated with at least 1 dose of study treatment and reported as AEs graded based on the National Cancer Institute Common Terminology Criteria for Adverse Events v3.0.

\section{Results}

\section{Patient population}

In total, 84 and 81 patients were randomized into the palbociclib plus letrozole arm and letrozole alone arm, respectively. Patient demographics and baseline disease characteristics were relatively balanced between the treatment arms (Table 1). Median age was similar in both treatment arms, and approximately half of the patients in each treatment arm had visceral metastases. Approximately half of the patients in each treatment arm had received prior adjuvant systemic treatment, with the most common treatments in both treatment arms being chemotherapy (41\% and $46 \%$ in the palbociclib plus letrozole and letrozole arms, respectively) and hormonal therapy (32\% and $36 \%$ in the palbociclib plus letrozole and letrozole arms, respectively). As of the data cutoff date (December 30, 2016), 80 patients in the palbociclib plus letrozole arm and 79 patients in the letrozole arm had permanently discontinued from the study. The most frequent reason for permanent discontinuation from the study treatment was objective disease progression $(63 \%$ and $77 \%$ of patients in the palbociclib plus letrozole and letrozole arms, respectively). Thirteen and 2 patients in the palbociclib plus letrozole and letrozole arms, respectively, permanently discontinued the study treatment due to AEs.

\section{Treatment exposure}

As of the data cutoff date, the median duration of follow-up was 64.7 months (palbociclib plus letrozole arm, 69.3 months; letrozole arm, 59.0 months). The median duration of treatment was 13.8 months (range, 0.2-77.0) in the palbociclib plus letrozole arm and 7.6 months (range, 0.9-64.7) in the letrozole arm. Mean relative dose intensity, defined as the actual dose divided by the intended dose, was $94 \%$ and $100 \%$ in the palbociclib plus letrozole and letrozole treatment arms, respectively. Thirty-four patients (41\%) in the palbociclib plus letrozole arm had the palbociclib dose reduced to $100 \mathrm{mg} /$ day, and 12 patients had the palbociclib dose further reduced to $75 \mathrm{mg} /$ day. Dosing interruptions due to AEs occurred in 30 patients $(36 \%)$, and cycle delays associated with AEs occurred in 38 patients (46\%) in the palbociclib plus letrozole arm. In the letrozole arm, 3 patients (3.9\%) had letrozole dosing interruption due to AEs.

\section{Efficacy}

Median OS was 37.5 months (95\% CI 31.4-47.8) in the palbociclib plus letrozole arm and 34.5 months (27.4-42.6) in the letrozole arm (stratified hazard ratio, $0.897 ; 95 \%$ CI $0.623-1.294 ; P=0.281$; Fig. 1a). OS was analyzed by subgroups based on baseline characteristics such as age, ECOG performance status, disease site, prior therapy, and DFI from the end of adjuvant treatment (Fig. 1b). Nonsignificant trends in favor of palbociclib plus letrozole were observed in most subgroups; however, the number of patients in each subgroup was small, and these data should be interpreted with caution.

\section{Subsequent treatments}

Most patients in both treatment arms received subsequent systemic therapy (83\% and $89 \%$ in the palbociclib plus letrozole and letrozole arms, respectively; Table 2). In the palbociclib plus letrozole arm, 38 (47.5\%), 13 (16.3\%), and 15 (18.8\%) patients received 1,2 , and $\geq 3$ subsequent regimens of therapy, respectively. In the letrozole arm, $29(36.7 \%), 11(13.9 \%)$, and $30(38.0 \%)$ patients received 1,2 , and $\geq 3$ subsequent regimens. The median (range) number of postprogression systemic therapies was 1 (1-6) and 2 (1-9) in the palbociclib plus letrozole and letrozole arms, respectively. The most frequent subsequent systemic therapy agent was hormonal therapy $(63 \%$ and 
Table 1 Patient demographics and baseline disease characteristics (ITT population)

\begin{tabular}{|c|c|c|}
\hline & PAL + LET $(n=84)$ & $\operatorname{LET}(n=81)$ \\
\hline Age, median (range), y & $63(41-89)$ & $64(38-84)$ \\
\hline \multicolumn{3}{|l|}{ Age distribution, years, $n(\%)$} \\
\hline $18-44$ & $2(2)$ & $4(5)$ \\
\hline $45-64$ & $45(54)$ & $38(47)$ \\
\hline$\geq 65$ & $37(44)$ & $39(48)$ \\
\hline \multicolumn{3}{|l|}{ Race, $n(\%)$} \\
\hline White & $76(90)$ & $72(89)$ \\
\hline Black & $1(1)$ & $1(1)$ \\
\hline Asian/other & $7(8)$ & $8(10)$ \\
\hline \multicolumn{3}{|l|}{ ECOG performance status, $n(\%)$} \\
\hline 0 & $46(55)$ & $45(56)$ \\
\hline 1 & $38(45)$ & $36(44)$ \\
\hline \multicolumn{3}{|l|}{ Disease stage, $n(\%)$} \\
\hline Stage III & $3(4)$ & $1(1)$ \\
\hline Stage IV & $81(96)$ & $80(99)$ \\
\hline \multicolumn{3}{|l|}{ Disease site, $n(\%)$} \\
\hline Visceral & $38(45)$ & $43(53)$ \\
\hline Bone only & $16(19)$ & $12(15)$ \\
\hline Other (nonvisceral) & $30(36)$ & $26(32)$ \\
\hline \multicolumn{3}{|l|}{ Disease-free interval, $n(\%)$} \\
\hline$>12$ months from adjuvant to recurrence & $25(30)$ & $30(37)$ \\
\hline $\begin{array}{l}\leq 12 \text { months from adjuvant to recurrence or de novo } \\
\text { advanced disease }\end{array}$ & $59(70)$ & $51(63)$ \\
\hline De novo advanced disease & $44(52)$ & $37(46)$ \\
\hline \multicolumn{3}{|l|}{ Prior systemic treatment, $n(\%)$} \\
\hline None & $44(52)$ & $37(46)$ \\
\hline Chemotherapy & $34(40)$ & $37(46)$ \\
\hline Antihormonal & $27(32)$ & $29(36)$ \\
\hline Tamoxifen & $24(29)$ & $25(31)$ \\
\hline Anastrozole & $8(10)$ & $12(15)$ \\
\hline Letrozole & $2(2)$ & $1(1)$ \\
\hline Exemestane & $4(5)$ & $2(2)$ \\
\hline
\end{tabular}

ECOG Eastern Cooperative Oncology Group, ITT intention-to-treat, LET letrozole, PAL palbociclib
$73 \%$ in the palbociclib plus letrozole and letrozole arms, respectively); the median (range) number of postprogression systemic hormonal therapies was 1 (1-3) and 1 (1-4), respectively. Fulvestrant was the most frequent subsequent hormonal therapy in both the palbociclib plus letrozole and letrozole arms (received by $34 \%$ and $43 \%$ of patients, respectively). Subsequent chemotherapy was used in 59\% of patients in the palbociclib plus letrozole arm and $65 \%$ of patients in the letrozole arm. The median (range) number of chemotherapy regimens received postprogression was $1(1-4)$ and $2(1-7)$ in the palbociclib plus letrozole and letrozole arms, respectively. Median time from randomization to first subsequent chemotherapy was longer in the palbociclib plus letrozole arm than in the letrozole arm (26.7 and 17.7 months, respectively; Fig. 2). Twelve patients $(15 \%)$ in the palbociclib plus letrozole arm and
13 patients (17\%) in the letrozole arm received subsequent mTOR inhibitor (everolimus). One and 2 patients in the palbociclib plus letrozole and letrozole alone arms, respectively, received a subsequent CDK4/6 inhibitor.

\section{Safety}

Consistent with previous reports, the most frequently reported all-causality AEs in the palbociclib plus letrozole arm were hematologic (neutropenia: any grade, $75 \%$; grade 3 or $4,59 \%$; leukopenia: any grade, $43 \%$; grade 3 or $4,18 \%$; Table 3). There were no reports of febrile neutropenia. The cumulative incidence of all-causality AEs reported by $>15 \%$ of patients during the first 5 years of treatment with palbociclib revealed that the incidence of AEs generally peaked 


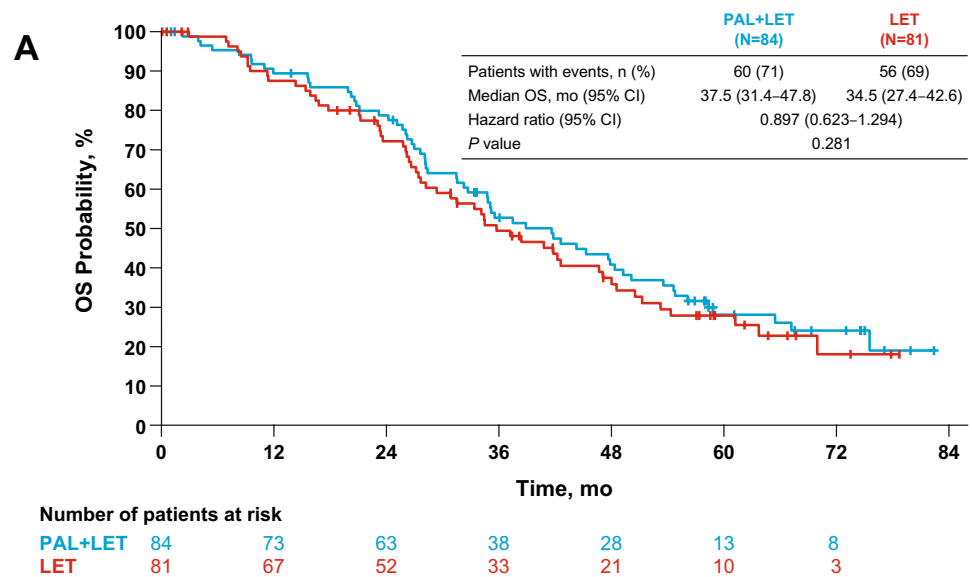

B

\begin{tabular}{llc}
\hline & Subgroup & $\mathbf{n}(\%)$ \\
\hline All randomized patients & & $165(100)$ \\
\hline \multirow{2}{*}{ Age } & $<65 \mathrm{y}$ & $89(54)$ \\
& $\geq 65 \mathrm{y}$ & $76(46)$ \\
\hline \multirow{2}{*}{ ECOG status } & 0 & $91(55)$ \\
& 1 & $74(45)$ \\
\hline \multirow{2}{*}{ Disease site } & Visceral & $81(49)$ \\
& Bone only & $28(17)$ \\
Prior chemotherapy & Other & $56(34)$ \\
\hline \multirow{2}{*}{ Prior hormonal therapy } & Yes & $71(43)$ \\
& No & $94(57)$ \\
\hline \multirow{2}{*}{ Prior systemic therapy } & Yes & $56(34)$ \\
& No & $109(66)$ \\
\hline \multirow{3}{*}{ Time from end of adjuvant } & Yes & $84(51)$ \\
treatment to disease & No & $81(49)$ \\
recurrence & $\leq 12$ mo or de novo & $110(67)$ \\
& $>12$ mo & $55(33)$ \\
\hline \multirow{2}{*}{ Region } & $\leq 12$ mo & $29(18)$ \\
& $>5 y$ & $58(35)$ \\
& $>5 y$ & $26(16)$ \\
\hline \multirow{2}{*}{ y y } & North America & $36(22)$ \\
& Europe & $123(75)$
\end{tabular}

Median OS $(95 \% \mathrm{Cl})$

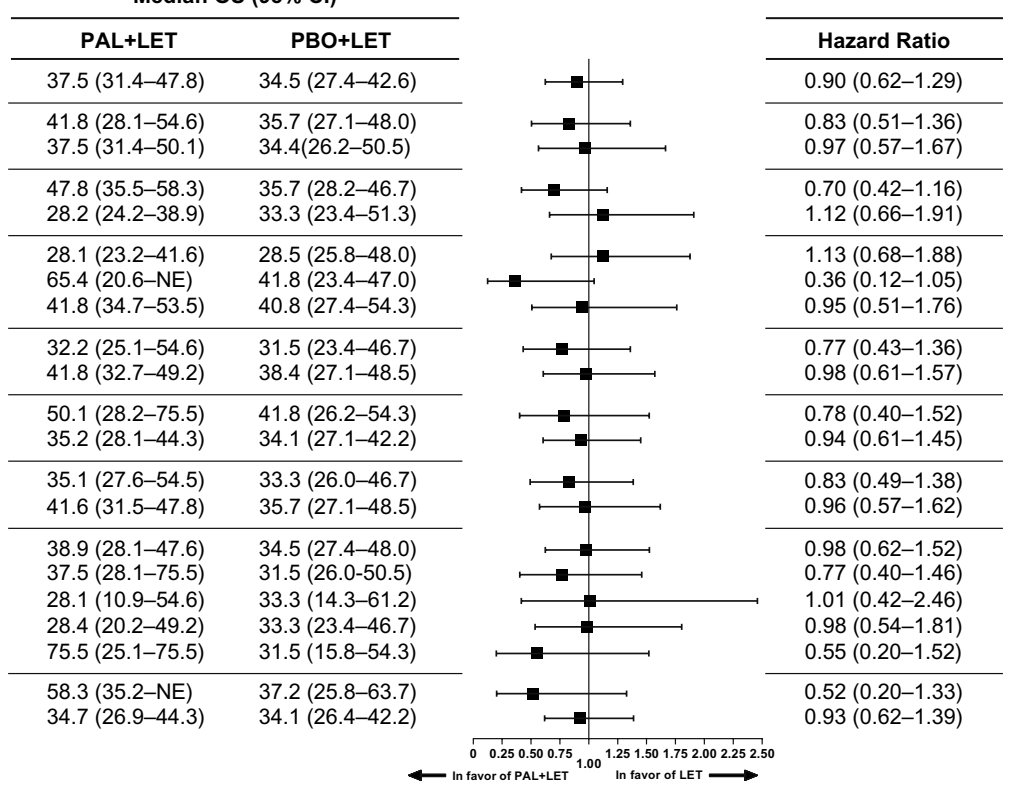

Fig. 1 Overall survival in the ITT population and by subgroup. a Kaplan-Meier curve of OS in the ITT population. b Forest plot of OS by subgroup. ECOG Eastern Cooperative Oncology Group, ITT

within the first year and then was relatively consistent over time (Fig. 3).

\section{Discussion}

The final OS analysis showed that OS was numerically longer in the palbociclib plus letrozole arm compared with the letrozole arm, although the results did not reach statistical significance. This trend was also observed for most subgroups; however, the number of patients in each subgroup was small. Based on previous analyses and given the longer median postprogression survival observed in both treatment arms, a larger sample size would likely be intention-to-treat, $L E T$ letrozole, $N E$ not estimable, $O S$ overall survival, $P A L$ palbociclib, $P B O$ placebo

needed to detect a statistically significant difference in OS in first-line ER+ breast cancer [12].

In addition, no new safety signals were observed in the current analysis; the incidence of AEs generally peaked within the first year and then remained constant for $\leq 5$ years of treatment with palbociclib. The most frequently reported all-causality AEs in the palbociclib plus letrozole arm were hematologic. These data demonstrated the consistent safety profile of palbociclib in combination with letrozole with long-term use and should provide confidence that there is no cumulative toxicity. These findings are in contrast to what is commonly seen with cytotoxic chemotherapies [14]; this is especially relevant as CDK4/6 
Table 2 Summary of subsequent anticancer treatment regimens

\begin{tabular}{lcc}
\hline & $\begin{array}{l}\text { PAL }+ \text { LET } \\
(n=80)\end{array}$ & LET $(n=79)$ \\
\hline Any systemic therapy, $n(\%)$ & $66(83)$ & $70(89)$ \\
Systemic therapy agents, $n(\%)$ & & \\
Antihormonal therapy & $50(63)$ & $58(73)$ \\
Nonsteroidal AI & $14(18)$ & $20(25)$ \\
Steroidal AI & $21(26)$ & $28(35)$ \\
Fulvestrant & $27(34)$ & $34(43)$ \\
Tamoxifen & $11(14)$ & $17(22)$ \\
Chemotherapy & $47(59)$ & $51(65)$ \\
Anthracyclines & $15(19)$ & $22(28)$ \\
Capecitabine & $27(34)$ & $33(42)$ \\
Gemcitabine & $4(5)$ & $8(10)$ \\
Taxanes & $34(43)$ & $31(39)$ \\
Vinorelbine & $12(15)$ & $6(8)$ \\
Other & $19(24)$ & $19(24)$ \\
mTOR inhibitor & $12(15)$ & $13(17)$ \\
Blinded therapy (clinical trial) & $2(3)$ & $5(6)$ \\
Palbociclib & $1(1)$ & $2(3)$ \\
\hline
\end{tabular}

$A I$ aromatase inhibitor, LET letrozole, mTOR mechanistic target of rapamycin, $P A L$ palbociclib

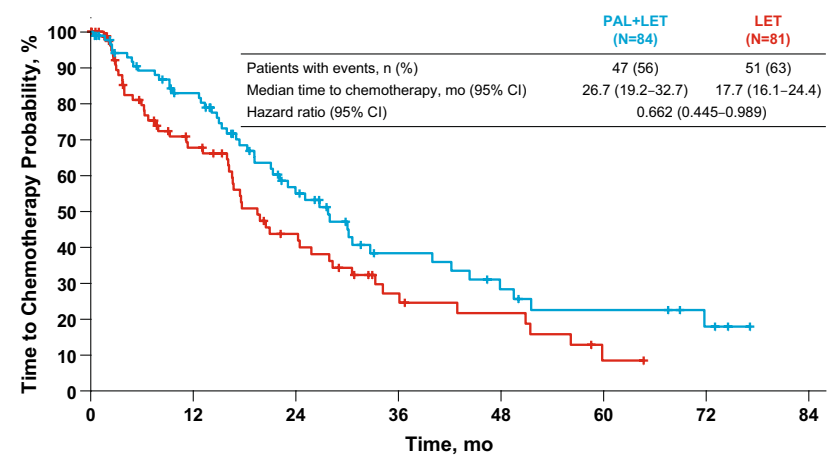

Fig. 2 Kaplan-Meier estimated time to first use of chemotherapy postprogression. $L E T$ letrozole, $P A L$ palbociclib

inhibitors are currently being evaluated in the early breast cancer setting [15].

Treatment with palbociclib plus letrozole prolonged the time to first use of chemotherapy compared with letrozole alone; delaying the use of cytotoxic chemotherapy can have a positive impact on patients' quality of life [13]. While the number of patients in each treatment arm who received subsequent systemic therapy was similar, fewer patients in the palbociclib plus letrozole arm versus the letrozole alone arm received $\geq 3$ subsequent systemic treatment regimens (18.8\% and $38.0 \%$, respectively). Patients in the letrozole alone arm received a median of 2 regimens of systemic therapy postprogression, driven by chemotherapy, compared with a median of 1 systemic postprogression therapy in the palbociclib plus letrozole arm. These differences could reflect the earlier time of first use of chemotherapy in the patients in the letrozole alone arm, and suggest that postprogression therapies may have impacted OS in this study. Since the results of PALOMA-1 were presented, substantial data have been generated with CDK4/6 inhibitors for the treatment of ABC, including OS data. To date, no other OS data have been presented from randomized studies of a CDK4/6 inhibitor in combination with an AI in postmenopausal women. Recently reported OS results from randomized studies showed prolonged OS with CDK4/6 inhibitors in combination with fulvestrant versus placebo plus fulvestrant [10, 16, 17]. As previously noted, a numerical but not statistically significantly longer OS was observed in the PALOMA-3 clinical trial, which evaluated the efficacy of palbociclib plus fulvestrant versus placebo plus fulvestrant for the treatment of patients with HR+/HER2- ABC whose disease had progressed on ET [10]. In MONALEESA-3, ribociclib plus fulvestrant was compared with placebo plus fulvestrant in the first- and second-line settings for postmenopausal women with HR+/HER2- ABC [16]. Ribociclib plus fulvestrant significantly prolonged OS versus placebo plus fulvestrant (hazard ratio, 0.724; 95\% CI 0.568-0.924; $P=0.00455$; median OS, not reached [NR] vs 40 months, respectively) [16]. MONARCH 2 compared abemaciclib plus fulvestrant versus placebo plus fulvestrant in women of any menopausal state (pre/perimenopausal women received ovarian suppression) with HR+/HER2- ABC whose disease had progressed on prior ET [17]. Abemaciclib plus fulvestrant prolonged OS compared with placebo plus fulvestrant (hazard ratio, $0.757 ; 95 \%$ CI $0.606-0.945 ; P=0.0137$; median OS, 46.7 vs 37.3 months, respectively) [17]. Of note, $33 \%$ of patients in PALOMA-3 received chemotherapy in the metastatic disease setting, whereas the MONARCH 2 and MONALEESA-3 trials did not allow prior chemotherapy for metastatic disease $[10,16,17]$. MONALEESA-7 evaluated ribociclib plus a nonsteroidal AI (NSAI) and ovarian suppression versus placebo plus ovarian suppression and an NSAI as first-line treatment in exclusively premenopausal women with $\mathrm{HR}+$ / HER2- ABC [18]. Ribociclib plus NSAI significantly prolonged OS versus placebo plus NSAI (hazard ratio, $0.71 ; 95 \%$ CI $0.54-0.95 ; P=0.00973$; median OS, NR vs 40.9 months, respectively) [18].

Since the initial readout of PALOMA-1, there have been 7 randomized phase 3 studies demonstrating the efficacy of CDK4/6 inhibition with ET versus ET alone [19-25]. These results confirm the importance of this target in ER+/HER2 - breast cancer and reinforce the findings seen in this smaller, randomized study, highlighting the success of a rational development program based on preclinical 


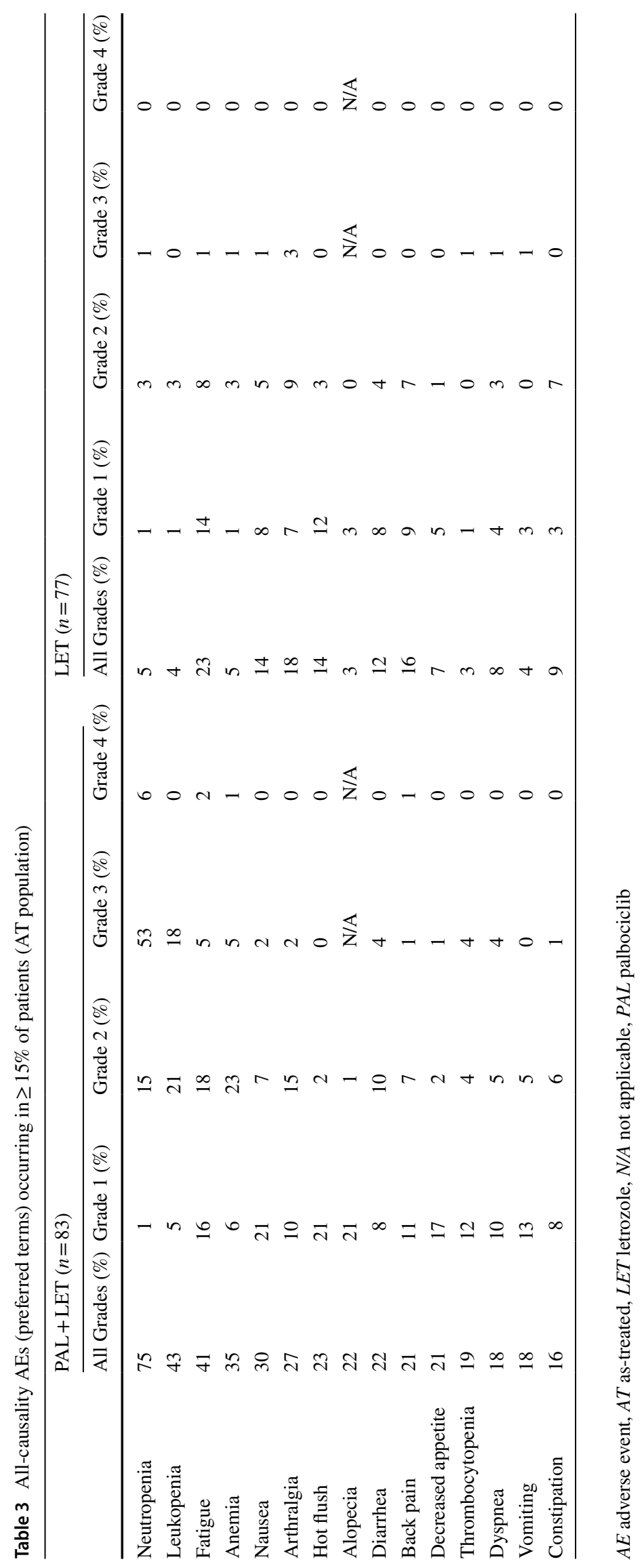



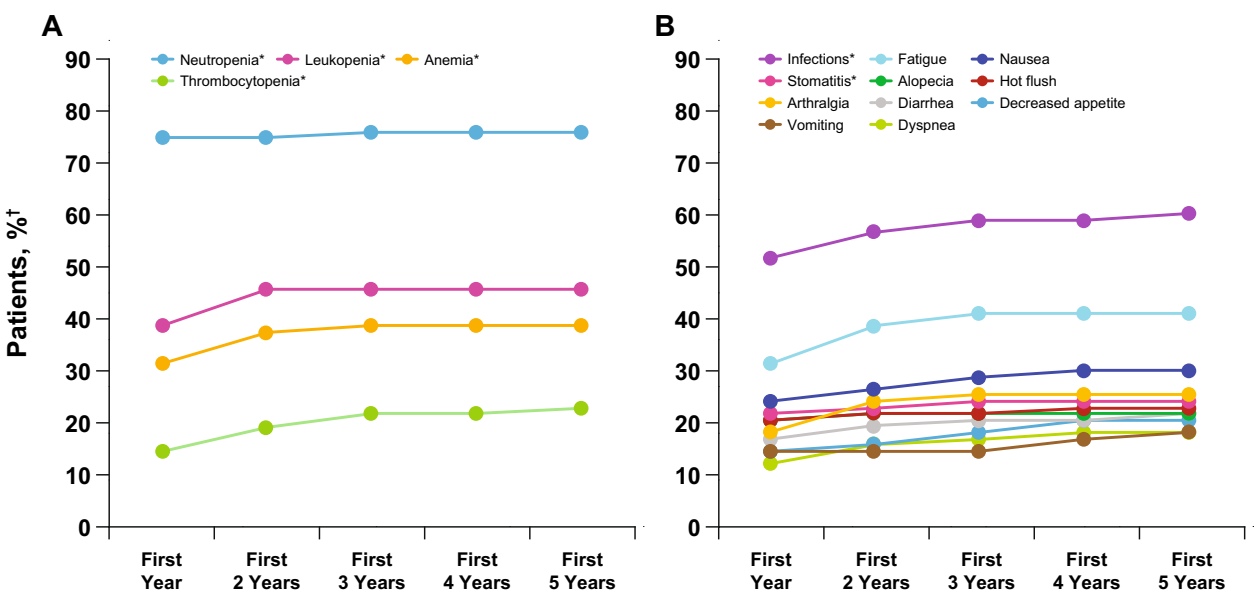

Fig. 3 Cumulative incidence of all-causality AEs $>15 \%$ during the first 5 years of treatment with palbociclib in the as-treated set. a Hematologic AEs. b Nonhematologic AEs. AEadverse event. *Grouped terms were as follows: neutropenia included the preferred terms neutropenia or neutrophil count decreased; anemia included the preferred terms anemia, hematocrit decreased, or hemoglobin decreased; leukopenia included the preferred terms leukopenia or

observations. Limitations of the PALOMA-1 trial include its open-label design and small sample size that may limit sufficient power to detect a statistically significant difference in OS; however, the long-term safety data reported here (median duration of follow-up of $>5$ years) and elsewhere [26] showed no cumulative toxicity of palbociclib plus ET and no new safety signals.

This report demonstrates a numerical increase in OS that is observed with the combination of palbociclib plus letrozole versus letrozole alone. These data, along with the published studies showing a statistically significant improvement in OS for patients receiving a CDK4/6 inhibitor in combination with various types of hormonal therapy, clearly support the use of CDK4/6 inhibitors in combination with ET as a standard of care for the treatment of patients with HR+/HER2- ABC. OS data from the phase 3 studies of NSAI in combination with CDK4/6 inhibitors in postmenopausal women, including the larger phase 3 PALOMA-2 study, are eagerly awaited.

Acknowledgements The authors thank all of the patients and their families, as well as all study investigators, research coordinators, and site staff. The authors also thank Camilla Fowst for her contribution to PALOMA-1. PALOMA-1 (NCT00721409) was sponsored by Pfizer Inc. Editorial support was provided by Jennifer Fetting, PhD, of ICON plc (North Wales, PA, USA), and was funded by Pfizer Inc.

Data availability Upon request, and subject to certain criteria, conditions, and exceptions (see https://www.pfizer.com/science/clini cal-trials/trial-data-and-results for more information), Pfizer will provide access to individual de-identified participant data from Pfizer-sponsored global interventional clinical studies conducted for white blood cell count decreased; infections included any preferred term that is part of the System Organ Class infections and infestations; and stomatitis included the preferred terms aphthous stomatitis, cheilitis, glossitis, glossodynia, mouth ulceration, mucosal inflammation, oral pain, oropharyngeal discomfort, oropharyngeal pain, or stomatitis. $†$ Patient percentage was calculated based on an $n=83$ denominator medicines, vaccines, and medical devices (1) for indications that have been approved in the US and/or EU or (2) in programs that have been terminated (i.e., development for all indications has been discontinued). Pfizer will also consider requests for the protocol, data dictionary, and statistical analysis plan. Data may be requested from Pfizer trials 24 months after study completion. The de-identified participant data will be made available to researchers whose proposals meet the research criteria and other conditions, and for which an exception does not apply, via a secure portal. To gain access, data requestors must enter into a data access agreement with Pfizer.

\section{Compliance with ethical standards}

Conflict of interest R.S. Finn has received consulting fees from Pfizer Inc, AstraZeneca, Bayer, Novartis, Bristol-Myers Squibb, Eisai, Eli Lilly, Merck, and Roche, as well as other research funding from Pfizer Inc and honoraria from Bayer, Pfizer Inc, Bristol-Myers Squibb, Novartis, Eisai, and Eli Lilly. K. Boer has received consulting fees from Pfizer Inc, Novartis, Eli Lilly, and Roche and served on speakers' bureaus for Pfizer Inc, Novartis, Eli Lilly, AstraZeneca, and Roche. I. Bondarenko, Y. Shparyk, A. Thummala, and N. Voitko have nothing to report. R. Patel is a stockholder of Novartis. J. Ettl received consulting fees from Eli Lilly, Novartis, Pfizer Inc, Roche, and Eisai; performed contracted research for Celgene; and received honoraria and travel support from Celgene, Eli Lilly, Novartis, Pfizer Inc, Roche, and Teva. T. Pinter has received consulting fees from Amgen and Pfizer Inc and served on speakers' bureaus for Roche, Bayer, Amgen, and Pfizer Inc. M. Schmidt has received honoraria and consulting fees from AstraZeneca, Celgene, Eisai, Eli Lilly, Myelo Therapeutics, Novartis, Pantarhei Oncology, Roche, Pfizer Inc, and Pierre Fabre; has received travel reimbursement from BioNTech, Pfizer Inc, and Roche; and has patents pending with Sividon. D.J. Slamon reports consultant/advisory roles for Pfizer Inc, Eli Lilly, and Novartis; stock ownership in Pfizer Inc; and research funding from Pfizer Inc and Novartis. E. Bananis, L. McRoy, K. Wilner, X. Huang, and S. Kim are employees and stockholders of Pfizer Inc. 
Ethical approval This clinical trial complied with the current laws of the countries in which it was performed.

Open Access This article is licensed under a Creative Commons Attribution 4.0 International License, which permits use, sharing, adaptation, distribution and reproduction in any medium or format, as long as you give appropriate credit to the original author(s) and the source, provide a link to the Creative Commons licence, and indicate if changes were made. The images or other third party material in this article are included in the article's Creative Commons licence, unless indicated otherwise in a credit line to the material. If material is not included in the article's Creative Commons licence and your intended use is not permitted by statutory regulation or exceeds the permitted use, you will need to obtain permission directly from the copyright holder. To view a copy of this licence, visit http://creativecommons.org/licenses/by/4.0/.

\section{References}

1. Bray F, Ferlay J, Soerjomataram I, Siegel RL, Torre LA, Jemal A (2018) Global cancer statistics 2018: GLOBOCAN estimates of incidence and mortality worldwide for 36 cancers in 185 countries. CA Cancer J Clin 68:394-424

2. American Cancer Society (2017) Breast cancer facts \& figures 2017-2018. https://www.cancer.org/content/dam/cancer-org/ research/cancer-facts-and-statistics/breast-cancer-facts-and-figur es/breast-cancer-facts-and-figures-2017-2018.pdf. Accessed 27 Aug 2019

3. Rugo HS, Rumble RB, Macrae E, Barton DL, Connolly HK, Dickler MN, Fallowfield L, Fowble B, Ingle JN, Jahanzeb M, Johnston SR, Korde LA, Khatcheressian JL, Mehta RS, Muss HB, Burstein HJ (2016) Endocrine therapy for hormone receptor-positive metastatic breast cancer: American Society of Clinical Oncology guideline. J Clin Oncol 34:3069-3103

4. Johnston SJ, Cheung K-L (2018) Endocrine therapy for breast cancer: a model of hormonal manipulation. Oncol Ther 6:141-156

5. National Comprehensive Cancer Network (2019) NCCN Clinical Practice Guidelines in Oncology (NCCN Guidelines ${ }^{\circledR}$ ). Breast Cancer Version 1.2019. https://www.ncen.org/professionals/physi cian_gls/default.aspx. Accessed 21 Feb 2020

6. Fry DW, Harvey PJ, Keller PR, Elliott WL, Meade M, Trachet E, Albassam M, Zheng X, Leopold WR, Pryer NK, Toogood PL (2004) Specific inhibition of cyclin-dependent kinase $4 / 6$ by PD 0332991 and associated antitumor activity in human tumor xenografts. Mol Cancer Ther 3:1427-1438

7. Finn RS, Dering J, Conklin D, Kalous O, Cohen DJ, Desai AJ, Ginther C, Atefi M, Chen I, Fowst C, Los G, Slamon DJ (2009) PD 0332991, a selective cyclin D kinase 4/6 inhibitor, preferentially inhibits proliferation of luminal estrogen receptor-positive human breast cancer cell lines in vitro. Breast Cancer Res 11:R77

8. Finn RS, Crown JP, Lang I, Boer K, Bondarenko IM, Kulyk SO, Ettl J, Patel R, Pinter T, Schmidt M, Shparyk Y, Thummala AR, Voytko NL, Fowst C, Huang X, Kim ST, Randolph S, Slamon DJ (2015) The cyclin-dependent kinase 4/6 inhibitor palbociclib in combination with letrozole versus letrozole alone as firstline treatment of oestrogen receptor-positive, HER2-negative, advanced breast cancer (PALOMA-1/TRIO-18): a randomised phase 2 study. Lancet Oncol 16:25-35

9. Rugo HS, Finn RS, Dieras V, Ettl J, Lipatov O, Joy AA, Harbeck $\mathrm{N}$, Castrellon A, Iyer S, Lu DR, Mori A, Gauthier ER, Bartlett CH, Gelmon KA, Slamon DJ (2019) Palbociclib plus letrozole as first-line therapy in estrogen receptor-positive/human epidermal growth factor receptor 2-negative advanced breast cancer with extended follow-up. Breast Cancer Res Treat 174:719-729

10. Turner NC, Slamon DJ, Ro J, Bondarenko I, Im SA, Masuda N, Colleoni M, DeMichele A, Loi S, Verma S, Iwata H, Harbeck N, Loibl S, Andre F, Puyana Theall K, Huang X, Giorgetti C, Huang Bartlett C, Cristofanilli M (2018) Overall survival with palbociclib and fulvestrant in advanced breast cancer. N Engl J Med 379:1926-1936

11. Ibrance ${ }^{\circledR}$ (palbociclib) (2018) Summary of product characteristics. Pfizer Limited, Sandwich, Kent, UK

12. Broglio KR, Berry DA (2009) Detecting an overall survival benefit that is derived from progression-free survival. J Natl Cancer Inst 101:1642-1649

13. Gupta S, Zhang J, Jerusalem G (2014) The association of chemotherapy versus hormonal therapy and health outcomes among patients with hormone receptor-positive, HER2-negative metastatic breast cancer: experience from the patient perspective. Expert Rev Pharmacoecon Outcomes Res 14:929-940

14. Partridge AH, Burstein HJ (2001) Winer EP (2001) Side effects of chemotherapy and combined chemohormonal therapy in women with early-stage breast cancer. J Natl Cancer Inst Monogr 30:135-142

15. Kwapisz D (2018) Cyclin-dependent kinase 4/6 inhibitors in hormone receptor-positive early breast cancer: preliminary results and ongoing studies. Breast Cancer 25:506-516

16. Slamon DJ, Neven P, Chia S, Fasching PA, De Laurentiis M, Im SA, Petrakova K, Bianchi GV, Esteva FJ, Martin M, Nusch A, Sonke GS, De la Cruz-Merino L, Beck JT, Pivot X, Sondhi M, Wang Y, Chakravartty A, Rodriguez-Lorenc K, Taran T, Jerusalem G (2020) Overall survival with ribociclib plus fulvestrant in advanced breast cancer. N Engl J Med 382:514-524

17. Sledge GW Jr, Toi M, Neven P, Sohn J, Inoue K, Pivot X, Burdaeva O, Okera M, Masuda N, Kaufman PA, Koh H, Grischke EM, Conte P, Lu Y, Barriga S, Hurt K, Frenzel M, Johnston S, Llombart-Cussac A (2020) The effect of abemaciclib plus fulvestrant on overall survival in hormone receptor-positive, ERBB2-negative breast cancer that progressed on endocrine therapy-MONARCH 2: a randomized clinical trial. JAMA Oncol 6:116-124

18. Im SA, Lu YS, Bardia A, Harbeck N, Colleoni M, Franke F, Chow L, Sohn J, Lee KS, Campos-Gomez S, Villanueva-Vazquez R, Jung KH, Chakravartty A, Hughes G, Gounaris I, RodriguezLorenc K, Taran T, Hurvitz S, Tripathy D (2019) Overall survival with ribociclib plus endocrine therapy in breast cancer. $\mathrm{N}$ Engl $\mathrm{J}$ Med 381:307-316

19. Finn RS, Martin M, Rugo HS, Jones S, Im SA, Gelmon K, Harbeck N, Lipatov ON, Walshe JM, Moulder S, Gauthier E, Lu DR, Randolph S, Dieras V, Slamon DJ (2016) Palbociclib and letrozole in advanced breast cancer. N Engl J Med 375:1925-1936

20. Turner NC, Ro J, Andre F, Loi S, Verma S, Iwata H, Harbeck N, Loibl S, Huang Bartlett C, Zhang K, Giorgetti C, Randolph S, Koehler M, Cristofanilli M, PALOMA3 Study Group (2015) Palbociclib in hormone-receptor-positive advanced breast cancer. N Engl J Med 373:209-219

21. Slamon DJ, Neven P, Chia S, Fasching PA, De Laurentiis M, Im SA, Petrakova K, Bianchi GV, Esteva FJ, Martin M, Nusch A, Sonke GS, De la Cruz-Merino L, Beck JT, Pivot X, Vidam G, Wang Y, Rodriguez Lorenc K, Miller M, Taran T, Jerusalem G (2018) Phase III randomized study of ribociclib and fulvestrant in hormone receptor-positive, human epidermal growth factor receptor 2-negative advanced breast cancer: MONALEESA-3. J Clin Oncol 36:2465-2472

22. Tripathy D, Im SA, Colleoni M, Franke F, Bardia A, Harbeck N, Hurvitz SA, Chow L, Sohn J, Lee KS, Campos-Gomez S, Villanueva Vazquez R, Jung KH, Babu KG, Wheatley-Price P, De Laurentiis M, Im YH, Kuemmel S, El-Saghir N, Liu MC, 
Carlson G, Hughes G, Diaz-Padilla I, Germa C, Hirawat S, Lu YS (2018) Ribociclib plus endocrine therapy for premenopausal women with hormone-receptor-positive, advanced breast cancer (MONALEESA-7): a randomised phase 3 trial. Lancet Oncol 19:904-915

23. Hortobagyi GN, Stemmer SM, Burris HA, Yap YS, Sonke GS, Paluch-Shimon S, Campone M, Blackwell KL, Andre F, Winer EP, Janni W, Verma S, Conte P, Arteaga CL, Cameron DA, Petrakova K, Hart LL, Villanueva C, Chan A, Jakobsen E, Nusch A, Burdaeva O, Grischke EM, Alba E, Wist E, Marschner N, Favret AM, Yardley D, Bachelot T, Tseng LM, Blau S, Xuan F, Souami F, Miller M, Germa C, Hirawat S, O'Shaughnessy J (2016) Ribociclib as first-line therapy for HR-positive, advanced breast cancer. N Engl J Med 375:1738-1748

24. Sledge GW Jr, Toi M, Neven P, Sohn J, Inoue K, Pivot X, Burdaeva O, Okera M, Masuda N, Kaufman PA, Koh H, Grischke EM, Frenzel M, Lin Y, Barriga S, Smith IC, Bourayou N, Llombart-Cussac A (2017) MONARCH 2: abemaciclib in combination with fulvestrant in women with HR+/HER2- advanced breast cancer who had progressed while receiving endocrine therapy. J Clin Oncol 35:2875-2884

25. Goetz MP, Toi M, Campone M, Sohn J, Paluch-Shimon S, Huober J, Park IH, Tredan O, Chen SC, Manso L, Freedman OC, Garnica Jaliffe G, Forrester T, Frenzel M, Barriga S, Smith IC, Bourayou N, Di Leo A (2017) MONARCH 3: abemaciclib as initial therapy for advanced breast cancer. J Clin Oncol 35:3638-3646

26. Diéras V, Rugo HS, Schnell P, Gelmon K, Cristofanilli M, Loi S, Colleoni M, Lu DR, Mori A, Gauthier E, Huang Bartlett C, Slamon DJ, Turner NC, Finn RS (2018) Long-term pooled safety analysis of palbociclib in combination with endocrine therapy for HR+/HER2- advanced breast cancer. J Natl Cancer Inst 111:419-430

Publisher's Note Springer Nature remains neutral with regard to jurisdictional claims in published maps and institutional affiliations.

\section{Affiliations}

\section{Richard S. Finn ${ }^{1}$ (D) Katalin Boer ${ }^{2} \cdot$ Igor Bondarenko ${ }^{3} \cdot$ Ravindranath Patel $^{4} \cdot$ Tamas Pinter $^{5} \cdot$ Marcus Schmidt $^{6}$. Yaroslav V. Shparyk ${ }^{7} \cdot$ Anu Thummala $^{8} \cdot$ Nataliia Voitko $^{9} \cdot$ Eustratios Bananis $^{10} \cdot$ Lynn McRoy $^{10} \cdot$ Keith Wilner $^{11}$. Xin Huang ${ }^{11}$. Sindy Kim ${ }^{11}$. Dennis J. Slamon ${ }^{1}$. Johannes Ettl ${ }^{12}$}

1 David Geffen School of Medicine, University of California Los Angeles, 2825 Santa Monica Blvd, Suite 200, Santa Monica, CA, USA

2 Onkologia, Szent Margit Korhaz, Budapest, Hungary

3 Dnipropetrovsk State Medical Academy, Dnipropetrovsk, Ukraine

4 Comprehensive Blood and Cancer Center, Bakersfield, CA, USA

5 Petz Aladar Megyei Oktato Korhaz, Gyor, Hungary

6 Department of Obstetrics and Gynecology, University Medical Center Mainz, Mainz, Germany
$7 \quad$ Lviv State Oncologic Regional Treatment and Diagnostic Center, Lviv, Ukraine

8 Comprehensive Cancer Centers of Nevada, Las Vegas, NV, USA

9 Kyiv City Clinical Oncology Center, Kyiv, Ukraine

10 Pfizer Inc, New York, NY, USA

11 Pfizer Inc, San Diego, CA, USA

12 Department of Obstetrics and Gynecology, Klinikum Rechts Der Isar, Technische Universität München, München, Germany 